\title{
Plantas medicinais no processo de cicatrização de feridas: uma revisão de literatura
}

\author{
PIRIZ, M.A. ${ }^{1 *}$; LIMA, C.A.B.'; JARDIM, V.M.R. ${ }^{1}$; MESQUITA, M.K. '; SOUZA, A.D.Z.'; HECK, R.M.1 \\ ${ }_{1}^{1}$ Programa de Pós-graduação em Enfermagem. Faculdade de Enfermagem. Universidade Federal de Pelotas. \\ Rua Gomes Carneiro, $n^{\circ} 1,2^{\circ}$ andar, sala 201. Centro, Pelotas, RS, Brasil, 96010-610. Telefone: (53)39211523. \\ ‘Autor correspondente: manuelle.piriz@gmail.com.
}

\begin{abstract}
RESUMO: Uma busca foi realizada na literatura, visando sumarizar as pesquisas realizadas e obter informações acerca da utilização de plantas medicinais no processo de cicatrização de feridas. Utilizaram-se os descritores: Plantas Medicinais e Cicatrização de Feridas e seus equivalentes em inglês e espanhol, com o operador booleano "AND" em três bases eletrônicas de dados (PubMed, LILACS e COCHRANE). Foram selecionados 57 artigos para compor a revisão. Os resultados apontam que um total de 52 plantas medicinais e um composto de ervas foram estudados experimentalmente ou clinicamente, quanto aos seus efeitos no auxílio do processo de cicatrização, sendo que a maioria $(88,5 \%)$ apresentou eficácia. Com isso, verificase que a utilização de plantas medicinais trata de importante alternativa no tratamento de feridas, que começa a fazer parte da atenção à saúde brasileira, o que sugere novos estudos de comprovação clínica, custos, e benefícios e a constante atualização acerca das publicações realizadas.
\end{abstract}

Palavras chave: extratos vegetais, fitoterapia, saúde pública, cicatrização.

\begin{abstract}
Medicinal plants in the wound healing process: a literature review. It was done a literature search aiming to sumarize earlier conducted researches and in order to obtain information on the use of medicinal plants in wound healing process. The descriptors used were as follows: Medicinal Plants and Wound Healing and their equivalents in English and Spanish with the Boolean operator "AND" in three electronic databases (PubMed, LILACS and COCHRANE). Fifty seven articles were chosen to compose the review. The findings from a total of fifty two medicinal plants and one herbal compound that were experimentally or clinically studied in respect to their effects in wound healing process indicate that the majority of them (eighty eight point five per cent) showed efficacy. Thus, it can be seen that the use of medicinal plants is an important alternative in wounds treatment that becomes part of Brazilian health care, suggesting further studies of clinical evidence, costs and benefits and constantly updating on produced publications.
\end{abstract}

Keywords: plant extracts, phytotherapy, public health, wound healing.

\section{INTRODUÇÃO}

O uso de plantas medicinais pode ser entendido como uma prática que atravessa milênios, estando historicamente presente na sabedoria do senso comum, articulando cultura e saúde, uma vez que estes aspectos não ocorrem isoladamente, mas inseridos em um contexto histórico determinado (Alvim et al, 2006).

Ao longo dos séculos, produtos de origem vegetal constituíram as bases para tratamento de diversas doenças, quer de forma tradicional, devido ao conhecimento das propriedades de determinada planta, que é passado de geração a geração, quer pela utilização de espécies vegetais, como fonte de moléculas ativas (Carvalho \& Silveira, 2010).

Recebido para publicação em 11/12/2012

Aceito para publicação em 24/03/2014
No processo de cicatrização de feridas, sua utilização não se difere, elas são mencionadas desde a pré-história, quando eram utilizadas plantas e extratos vegetais, na forma de cataplasmas, com o intuito de estancar hemorragias e favorecer a cicatrização, sendo muitas dessas plantas ingeridas, para atuação em via sistêmica (Silva \& Mocelin, 2007).

Com isso, pode-se afirmar que a medicina, como conhecemos hoje, só foi possível pelo resgate acerca dos métodos de cura e conhecimentos empíricos utilizados há milhares de anos (Aquino et al, 2007). Assim, atualmente, existem diversos recursos disponíveis para auxiliar no processo de cicatrização e sua aplicação na realização de

Rev. Bras. PI. Med., Campinas, v.16, n.3, p.628-636, 2014. 
curativos e técnicas para o tratamento de feridas (Mandelbaum et al, 2003).

Neste sentido, é importante lembrar que - Ministério da Saúde brasileiro, nos últimos anos, busca estimular a inserção das práticas complementares de cuidado no sistema oficial de saúde. Destaca-se a implementação da Política Nacional de Plantas Medicinais e Fitoterápicos (PNPMF) (Brasil, 2006a) e a Política Nacional de Práticas Integrativas e Complementares (PNPIC) (Brasil, 2006b), ambas no ano de 2006, e que visam estimular o acesso às práticas complementares e às plantas medicinais, para o cuidado em saúde, de forma eficaz e segura.

Outras publicações importantes são a Relação Nacional de Plantas Medicinais de interesse ao Sistema Único de Saúde (SUS), lançada em 2009 , contendo 71 plantas medicinais que devem ser objeto de pesquisa e implementação dos setores e serviços de saúde públicos brasileiros (Brasil, 2009). Já a Resolução da Diretoria Colegiada (RDC) $n^{\circ}$ 10 , do ano de 2010, lista 66 plantas medicinais com comprovadas ações na saúde humana; dentre estas, várias espécies são indicadas para o processo de cicatrização, o que implica em grande avanço da saúde pública brasileira que começa a valorizar a utilização de novas terapias baseadas nas plantas medicinais, prática tão difundida ao redor do mundo e das gerações.

Considerando a importância de realizar buscas que reafirmem os benefícios e comprovações de eficácia das plantas medicinais, o objetivo deste estudo foi identificar quais plantas medicinais vêm sendo empregadas no processo de cicatrização de feridas ao redor do mundo, e quais estudos têm identificado suas ações.

\section{Caminho metodológico}

O presente estudo trata-se de uma análise de dados secundários, por meio de uma revisão sistemática da literatura (Galvão et al, 2004), que objetivou responder à seguinte questão: "Quais plantas medicinais estão sendo pesquisadas para o processo de cicatrização de feridas ao redor do mundo, e quais estudos científicos de comprovação foram realizados nos últimos 20 anos?".
As buscas foram realizadas de maio a junho de 2012, nas bases eletrônicas de dados LILACS, PubMed e COCHRANE, utilizando os descritores previamente consultados no DECs (Descritores em Ciências da Saúde) "Medicinal plants" AND "Wound healing" e seus correspondentes em português, "Plantas medicinais" e "Cicatrização de feridas" e, em espanhol, "Plantas medicinales" $Y$ "Cicatrización de heridas".

Como limite, foi utilizado o período de tempo de 1992 a 2012 (20 anos), na base PubMed; nas demais bases, o ano que ultrapassasse o limite foi utilizado como critério de exclusão. Após nova análise para atualização, realizou-se novamente a busca, incluindo o ano de 2013 como critério de busca.

Foram incluídos, após leitura de títulos e resumos, os artigos que se encaixavam no tema proposto e discutiam plantas medicinais no processo de cicatrização de feridas, por meio de estudos de comprovação, redigidos nas línguas inglesa, portuguesa ou espanhola, enfocando primordialmente estudos experimentais, in vitro ou de cunho clínico randomizado.

Como critérios de exclusão, adotaram-se artigos que não apresentassem nenhum aspecto do tema proposto ou que estivessem redigidos em outras línguas que não as acima citadas ou trouxessem resultados de teses e dissertações, devido ao grande tamanho destas publicações.

\section{RESULTADOS ENCONTRADOS}

O quadro 1 mostra os resultados encontrados em cada uma das bases de dados pesquisada.

Dessa forma, foram selecionados 46 artigos de estudos científicos com plantas medicinais na cicatrização, para apresentação no presente trabalho, e mais 11 artigos foram acrescentados à busca, devido à sua relevância científica e benefícios para o conhecimento do potencial de plantas nativas brasileiras, totalizando 57 artigos.

A seguir, no quadro 2, apresentam-se os estudos realizados ao redor do mundo, nos últimos 20 anos, e que tiveram por objetivo testar cientificamente o extrato das plantas medicinais,

Quadro 1. Resultado das buscas e caminho metodológico nas bases PubMed, LILACS e COCHRANE, Brasil, 2013.

\begin{tabular}{ccccc}
\hline $\begin{array}{c}\text { Base de } \\
\text { dados }\end{array}$ & $\begin{array}{c}\text { Total de } \\
\text { artigos }\end{array}$ & $\begin{array}{c}\text { Excluídos após leitura de } \\
\text { títulos e resumos }\end{array}$ & $\begin{array}{c}\text { Lidos na } \\
\text { íntegra }\end{array}$ & Selecionados \\
\hline PubMed & 81 & 37 & 44 & 33 \\
LILACS & 26 & 15 & 11 & 11 \\
COCHRANE & 15 & 13 & 02 & 02 \\
Total & $\mathbf{1 2 2}$ & $\mathbf{6 5}$ & $\mathbf{5 7}$ & 46 \\
\hline
\end{tabular}

Rev. Bras. PI. Med., Campinas, v.16, n.3, p.628-636, 2014. 
Quadro 2. Estudos clínicos e experimentais com plantas medicinais na cicatrização de feridas. Brasil. 2013.

\begin{tabular}{|c|c|c|c|c|c|c|}
\hline Autores & Ano & País & $\begin{array}{l}\text { Tipo de } \\
\text { estudo }\end{array}$ & Sujeitos & Planta(s) pesquisadas & Modo de aplicação \\
\hline Davis et al. & 1994 & $\begin{array}{l}\text { Estados } \\
\text { Unidos }\end{array}$ & $\begin{array}{c}\text { Estudo } \\
\text { Experimental }\end{array}$ & Ratos & Aloe vera (L.) Burm. f. & Utilizada topicamente \\
\hline $\begin{array}{l}\text { Nuzov, } \\
\text { Stadinikov }\end{array}$ & 1994 & Rússia & $\begin{array}{l}\text { Estudo } \\
\text { Experimental }\end{array}$ & 73 ratos & $\begin{array}{l}\text { Óleo Miliaceum (produtos } \\
\text { processados do milho) }\end{array}$ & Aplicado topicamente \\
\hline Davis et al. & 1994 & $\begin{array}{l}\text { Estados } \\
\text { Unidos }\end{array}$ & $\begin{array}{l}\text { Estudo } \\
\text { Experimental }\end{array}$ & $\begin{array}{l}15 \text { ratos } \\
\text { machos } \\
\text { adultos }\end{array}$ & Aloe Vera (L.) Burm. f. & Aplicado topicamente \\
\hline Visuthikosol & 1995 & Tailândia & $\begin{array}{l}\text { Estudo clínico } \\
\text { Randomizado }\end{array}$ & $\begin{array}{l}27 \text { pacientes } \\
\text { com } \\
\text { queimaduras } \\
\text { parciais } \\
\end{array}$ & Aloe vera (L.) Burm. f. & $\begin{array}{c}\text { Gel de Aloe vera aplicado } \\
\text { topicamente. }\end{array}$ \\
\hline Sanchéz et al. & 1995 & Brasil & $\begin{array}{l}\text { Estudo } \\
\text { Experimental }\end{array}$ & 10 coelhos & Calendula officinalis L. & $\begin{array}{l}\text { Extrato alcoólico de 30c } \\
\text { Calendula officinalis diluídas } \\
\text { em } 50 \text { por cento de água. }\end{array}$ \\
\hline Morisaki et al. & 1995 & Japão & $\begin{array}{c}\text { Estudo } \\
\text { Experimental }\end{array}$ & $\begin{array}{c}\text { Ratos } \\
\text { diabéticos }\end{array}$ & Ginseng rubra Radix & $\begin{array}{c}\text { A saponina do Ginseng } \\
\text { utilizada topicamente em } \\
\text { feridas. }\end{array}$ \\
\hline Roberts, Travis & 1995 & $\begin{array}{l}\text { Estados } \\
\text { Unidos }\end{array}$ & $\begin{array}{c}\text { Estudo } \\
\text { Experimental }\end{array}$ & Ratos machos & Aloe vera (L.) Burm. f. & Gel aplicado topicamente. \\
\hline Castro e Silva & 1996 & Brasil & $\begin{array}{c}\text { Estudo } \\
\text { experimental }\end{array}$ & $\begin{array}{c}8 \text { ratos albinos } \\
\text { wistar }\end{array}$ & $\begin{array}{l}\text { Stryphnodendron } \\
\text { barbatiman (Mart). }\end{array}$ & Extrato da planta. \\
\hline Camargo et al. & 1996 & Brasil & $\begin{array}{l}\text { Estudo } \\
\text { Experimental }\end{array}$ & $\begin{array}{l}\text { Camundongos } \\
\text { tipo switz }\end{array}$ & Casearia sylvestris Sw. & $\begin{array}{c}\text { Inoculado o extrato } \\
\text { alcoólico tamponado da } \\
\text { planta no subcutâneo dos } \\
\text { camundongos. }\end{array}$ \\
\hline Suguna et al. & 1996 & Índia & $\begin{array}{c}\text { Estudo } \\
\text { Experimental }\end{array}$ & Ratos & Centella asiática (L.) Urban & $\begin{array}{c}\text { Extrato alcoólico de } C \text {. } \\
\text { asiatica em ratos por via } \\
\text { tópica e oral. }\end{array}$ \\
\hline Heggers et al. & 1996 & $\begin{array}{l}\text { Estados } \\
\text { Unidos }\end{array}$ & $\begin{array}{c}\text { Estudo } \\
\text { Experimental }\end{array}$ & $\begin{array}{l}\text { Ratos } \\
\text { Sprague- } \\
\text { Dawley } \\
\end{array}$ & Aloe vera (L.) Burm. f. & $\begin{array}{l}\text { Extrato da planta aplicado } \\
\text { topicamente }\end{array}$ \\
\hline Heggers et al. & 1997 & $\begin{array}{l}\text { Estados } \\
\text { Unidos }\end{array}$ & $\begin{array}{c}\text { Estudo } \\
\text { Experimental }\end{array}$ & $\begin{array}{c}\text { Ratos } \\
\text { Sprague-Dawle }\end{array}$ & Aloe vera (L.) Burm. f." & $\begin{array}{c}\text { Utilizada topicamente } \\
\text { associada à nitroglicerina e } \\
\text { L-NAME }\end{array}$ \\
\hline Saha et al. & 1997 & Índia & $\begin{array}{c}\text { Estudo } \\
\text { Experimental }\end{array}$ & $\begin{array}{c}6 \text { ratos wistar } \\
\text { albinos machos }\end{array}$ & $\begin{array}{c}\text { Leucas lavandulaefolia } \\
\text { Rees. }\end{array}$ & $\begin{array}{l}\text { Extrato metanólico da planta } \\
\text { sob a forma de pomada e } \\
\text { injeção. Ambos com efeito } \\
\text { positivo. }\end{array}$ \\
\hline Brito et al. & 1998 & Brasil & $\begin{array}{c}\text { Estudo } \\
\text { Experimental }\end{array}$ & $\begin{array}{l}60 \text { ratos } \\
\text { machos } \\
\text { adultos }\end{array}$ & Copaíba" & Óleo de copaíba puro. \\
\hline Chithra et al. & 1998 & Índia & $\begin{array}{c}\text { Estudo } \\
\text { Experimental }\end{array}$ & Ratos & Aloe Vera (L.) Burm. f. & $\begin{array}{c}\text { Aplicada topicamente em } \\
\text { ratos. }\end{array}$ \\
\hline $\begin{array}{l}\text { Sunilkumar } \\
\text { et al. }\end{array}$ & 1998 & Índia & $\begin{array}{c}\text { Estudo } \\
\text { Experimental }\end{array}$ & Ratos & Centella asiatica (L.) Urban & $\begin{array}{l}\text { Creme, pomada ou gel feitos } \\
\text { com a planta aplicados } \\
\text { topicamente. O gel mostrou- } \\
\text { se mais efetivo. }\end{array}$ \\
\hline
\end{tabular}

continua...

Rev. Bras. PI. Med., Campinas, v.16, n.3, p.628-636, 2014. 
Quadro 2. Estudos clínicos e experimentais com plantas medicinais na cicatrização de feridas. Brasil. 2013.

\begin{tabular}{|c|c|c|c|c|c|c|}
\hline Chithra et al. & 1998 & Índia & $\begin{array}{c}\text { Estudo } \\
\text { Experimental }\end{array}$ & $\begin{array}{l}\text { Ratos wistar } \\
\text { machos }\end{array}$ & Aloe vera (L.) Burm. f. & $\begin{array}{c}\text { Formulações de uso tópico e } \\
\text { oral de Aloe vera. Ambas com } \\
\text { efeitos semelhantes. }\end{array}$ \\
\hline Chithra et al. & 1998 & Índia & $\begin{array}{c}\text { Estudo } \\
\text { Experimental }\end{array}$ & $\begin{array}{c}\text { Ratos wistar } \\
\text { machos }\end{array}$ & Aloe Vera (L.) Burm. f. & $\begin{array}{l}\text { Gel da planta administrado } \\
\text { topicamente e por via oral. }\end{array}$ \\
\hline Chithra et al. & 1998 & Índia & $\begin{array}{c}\text { Estudo } \\
\text { Experimental }\end{array}$ & Ratos & Aloe vera (L.) Burm f. & $\begin{array}{c}\text { Ambos os preparados tópico } \\
\text { e oral. }\end{array}$ \\
\hline Brito et al. & 1999 & Brasil & $\begin{array}{c}\text { Estudo } \\
\text { experimental }\end{array}$ & $\begin{array}{l}60 \text { ratos } \\
\text { machos } \\
\text { adultos }\end{array}$ & Copaíba & Óleo de copaíba puro \\
\hline Maquart et al. & 1999 & França & $\begin{array}{c}\text { Estudo } \\
\text { Experimental }\end{array}$ & Ratos & Centella asiática (L.) Urban & $\begin{array}{c}\text { Mistura reconstituída, } \\
\text { utilizando triterpenos } \\
\text { extraídos a partir da planta, } \\
\text { o ácido asiático, ácido } \\
\text { madecassic e asiaticoside. } \\
\text { Injetável. }\end{array}$ \\
\hline $\begin{array}{l}\text { Mukherjee } \\
\text { et al. }\end{array}$ & 2000 & Índia & $\begin{array}{c}\text { Estudo } \\
\text { Experimental }\end{array}$ & Ratos & $\begin{array}{c}\text { Hypericum patulum Wall. ex } \\
\text { Dyer. }\end{array}$ & $\begin{array}{c}\text { Extrato metanólico sob a } \\
\text { forma de pomada com duas } \\
\text { concentrações diferentes ( } 5 \% \\
\text { e } 10 \% \text { ) }\end{array}$ \\
\hline $\begin{array}{c}\text { Somboonwong } \\
\text { et al. }\end{array}$ & 2000 & Tailândia & $\begin{array}{c}\text { Estudo } \\
\text { Experimental }\end{array}$ & $\begin{array}{c}48 \text { ratos wistar } \\
\text { machos }\end{array}$ & Aloe vera (L.) Burm. f. & $\begin{array}{c}\text { Gel liofilizado da planta, } \\
\text { usado topicamente }\end{array}$ \\
\hline $\begin{array}{l}\text { Mukherjee, } \\
\text { Suresh }\end{array}$ & 2000 & Índia & $\begin{array}{c}\text { Estudo } \\
\text { Experimental }\end{array}$ & $\begin{array}{l}72 \text { ratos } \\
\text { brancos de } \\
\text { ambos os } \\
\text { sexos }\end{array}$ & $\begin{array}{c}\text { Hypericum hookerianum } \\
\text { Leaf. }\end{array}$ & $\begin{array}{l}\text { Extrato metanólico das folhas } \\
\text { e caule sob a forma de } \\
\text { pomada de uso tópico. }\end{array}$ \\
\hline $\begin{array}{l}\text { Dominguez } \\
\text { et al. }\end{array}$ & 2001 & Cuba & $\begin{array}{c}\text { Estudo } \\
\text { experimental }\end{array}$ & $\begin{array}{c}24 \text { ratos } \\
\text { albinos machos }\end{array}$ & $\begin{array}{c}\text { Bryophyllum pinnata (Lam.) } \\
\text { Oken. }\end{array}$ & Extrato fluido da planta. \\
\hline $\begin{array}{l}\text { Nagappa, } \\
\text { Cheriyan }\end{array}$ & 2001 & India & $\begin{array}{c}\text { Estudo } \\
\text { Experimental }\end{array}$ & $\begin{array}{l}\text { Ratos wistar } \\
\text { de ambos os } \\
\text { sexos }\end{array}$ & $\begin{array}{c}\text { Thespesia } \\
\text { Populnea (L.) Sol. ex } \\
\text { Corrêa. }\end{array}$ & Extrato aquoso da fruta \\
\hline $\begin{array}{c}\text { Park \& Chun, } \\
2001\end{array}$ & 2001 & $\begin{array}{l}\text { Coréia do } \\
\text { Sul }\end{array}$ & $\begin{array}{c}\text { Estudo } \\
\text { experimental }\end{array}$ & \begin{tabular}{|c|} 
Ratos \\
Sprague- \\
Dawley fêmeas
\end{tabular} & Opuntia ficus-indica Mill. & $\begin{array}{c}\text { Extrato metanólico } \\
\text { administrado topicamente }\end{array}$ \\
\hline $\begin{array}{l}\text { Melo Junior } \\
\text { et al. }\end{array}$ & 2002 & Brasil & $\begin{array}{c}\text { Estudo } \\
\text { experimental }\end{array}$ & $\begin{array}{c}34 \text { ratos } \\
\text { winstar albinos } \\
\text { machos }\end{array}$ & Schinus terebinthifolis Raddi & Extrato da planta. \\
\hline $\begin{array}{l}\text { Martinéz } \\
\text { Fernandes } \\
\text { et al. }\end{array}$ & 2003 & Cuba & $\begin{array}{c}\text { Estudo } \\
\text { experimental }\end{array}$ & 40 ratos & Bidens Alba (L.) DC. & $\begin{array}{c}\text { Extrato fluido com } 4,5 \text { por } \\
\text { cento de sólidos totais de } \\
\text { Bidens Alba. }\end{array}$ \\
\hline $\begin{array}{l}\text { Oladejo et al., } \\
\qquad 2003\end{array}$ & 2003 & África & $\begin{array}{c}\text { Estudo } \\
\text { experimental }\end{array}$ & $\begin{array}{l}30 \text { ratos } \\
\text { winstar }\end{array}$ & $\begin{array}{l}\text { Ageratum conyzoides L. } \\
\text { comparado com o mel }\end{array}$ & $\begin{array}{l}\text { Gaze embebida no extrato da } \\
\text { planta. }\end{array}$ \\
\hline $\begin{array}{c}\text { Tillán Capo } \\
\text { et al. } \\
\end{array}$ & 2004 & Cuba & $\begin{array}{c}\text { Estudo } \\
\text { experimental }\end{array}$ & $\begin{array}{l}30 \text { ratos } \\
\text { machos }\end{array}$ & Cera de Cana de açúcar & $\begin{array}{c}\text { Extrato hidroalcoólico da } \\
\text { planta. }\end{array}$ \\
\hline Guillermo et al. & 2005 & Peru & $\begin{array}{c}\text { Estudo } \\
\text { experimental }\end{array}$ & $\begin{array}{c}96 \text { ratos } \\
\text { albinos machos }\end{array}$ & $\begin{array}{c}\text { Peperomia scutellaefolia } \\
\text { Ruiz \& Pav. }\end{array}$ & $\begin{array}{l}\text { Extrato hidroalcoólico da } \\
\text { planta em forma de gel. }\end{array}$ \\
\hline
\end{tabular}


Quadro 2. Estudos clínicos e experimentais com plantas medicinais na cicatrização de feridas. Brasil. 2013.

continuação...

\begin{tabular}{|c|c|c|c|c|c|c|}
\hline $\begin{array}{c}\text { Cavalcante } \\
\text { et al. }\end{array}$ & 2005 & Brasil & $\begin{array}{c}\text { Estudo } \\
\text { experimental }\end{array}$ & 48 ratos Wistar & $\begin{array}{c}\text { Myracrodruon urundeuva } \\
\text { fr. All. }\end{array}$ & $\begin{array}{l}\text { Extrato aquoso de aroeira a } \\
10 \% \text { aplicado topicamente. }\end{array}$ \\
\hline $\begin{array}{c}\text { González- } \\
\text { Quevedo et al. }\end{array}$ & 2006 & Cuba & $\begin{array}{c}\text { Estudo } \\
\text { experimental }\end{array}$ & 78 ratos & $\begin{array}{c}\text { Pinus } \\
\text { caribaea Morelet } \\
\end{array}$ & $\begin{array}{c}\text { Creme concentrado a } 5 \% \text { com } \\
\text { extrato da planta. }\end{array}$ \\
\hline $\begin{array}{l}\text { Moura-Letts } \\
\text { et al. }\end{array}$ & 2006 & Peru & $\begin{array}{c}\text { Estudo } \\
\text { experimental }\end{array}$ & Ratos albinos & $\begin{array}{l}\text { Anredera difusa (Moq.) } \\
\text { Sperling. }\end{array}$ & $\begin{array}{c}40 \text { mcg de ácido oleanólico } \\
\text { extraído da planta por grama } \\
\text { de peso. }\end{array}$ \\
\hline Chah et al. & 2006 & Nigéria & $\begin{array}{l}\text { Estudo } \\
\text { experimental }\end{array}$ & $\begin{array}{l}30 \text { ratos } \\
\text { albinos de } \\
\text { ambos os } \\
\text { sexos }\end{array}$ & $\begin{array}{c}\text { Ageratum conyzoide L., } \\
\text { Anthocleista djalonensis A. } \\
\text { Chev., Napoleona imperialis } \\
\text { P. Beauv., Ocimum } \\
\text { Gratissimum L., } \\
\text { Psidium guajava L. }\end{array}$ & Extratos metanólicos \\
\hline Servin et al. & 2006 & Brasil & $\begin{array}{c}\text { Estudo } \\
\text { experimental }\end{array}$ & 40 ratos Wistar & Atropha gossypiifolia L. & $\begin{array}{c}\text { Extrato hidroalcoólico por via } \\
\text { intraperitonial }\end{array}$ \\
\hline $\begin{array}{l}\text { Nayak \& } \\
\text { Mohan }\end{array}$ & 2007 & $\begin{array}{l}\text { Trinidad } \\
\text { and } \\
\text { Tobago }\end{array}$ & $\begin{array}{c}\text { Estudo } \\
\text { experimental }\end{array}$ & $\begin{array}{c}30 \text { ratos } \\
\text { Wistar albinos } \\
\text { machos }\end{array}$ & $\begin{array}{c}\text { Jasminum grandflorum Linn } \\
\text { Flower. }\end{array}$ & $\begin{array}{c}\text { Extrato etanólico administrado } \\
\text { por via oral das flores de } \\
\text { Jasmim. }\end{array}$ \\
\hline Parente et al. & 2009 & Brasil & $\begin{array}{c}\text { Estudo } \\
\text { experimental }\end{array}$ & $\begin{array}{l}36 \text { ratos Wistar } \\
\text { fêmeas }\end{array}$ & Calendula officinalis L. & $\begin{array}{l}\text { Solução aquosa do extrato } \\
\text { etanólico a } 1 \% \text {, topicamente. }\end{array}$ \\
\hline $\begin{array}{l}\text { Silva et al., } \\
2010\end{array}$ & 2010 & Brasil & $\begin{array}{c}\text { Estudo } \\
\text { experimental }\end{array}$ & $\begin{array}{c}40 \text { ratos tipo } \\
\text { Wistar machos }\end{array}$ & $\begin{array}{c}\text { Pfaffia glomerata. (Spreng.) } \\
\text { Pedersen }\end{array}$ & $\begin{array}{c}\text { Extrato de Pfaffia glomerata, } \\
\text { tópico. }\end{array}$ \\
\hline Li et al. & 2011 & China & $\begin{array}{c}\text { Ensaio } \\
\text { Clínico } \\
\text { Randomizado }\end{array}$ & $\begin{array}{l}57 \text { pacientes } \\
\text { portadores } \\
\text { de úlceras } \\
\text { crônicas de pé } \\
\text { diabético }\end{array}$ & $\begin{array}{c}\text { Composto de ervas } \\
\text { chinesas Pomada Tangzu } \\
\text { Yuyang }\end{array}$ & Pomada tópica das ervas \\
\hline Atiba et al. & 2011 & Japão & $\begin{array}{c}\text { Estudo } \\
\text { experimental }\end{array}$ & $\begin{array}{c}\text { Ratos } \\
\text { diabéticos } \\
\text { tipo } 2\end{array}$ & Aloe Vera (L.) Burm. f. & $\begin{array}{c}\text { Administração oral de Aloe } \\
\text { vera. }\end{array}$ \\
\hline Prichoa et al. & 2011 & Brasil & $\begin{array}{c}\text { Estudo } \\
\text { experimental }\end{array}$ & $\begin{array}{c}36 \text { ratos Wistar } \\
\text { machos }\end{array}$ & Sonchus oleraceus L. & $\begin{array}{l}\text { Extrato etanólico a } 10 \% \\
\text { aplicado topicamente. }\end{array}$ \\
\hline Parente et al. & 2011 & Brasil & $\begin{array}{c}\text { Estudo } \\
\text { experimental }\end{array}$ & $\begin{array}{c}12 \text { ratos Wistar } \\
\text { fêmeas }\end{array}$ & Calendula officinalis L. & $\begin{array}{l}\text { Extrato hidro alcoólico } \\
\text { aplicado topicamente }\end{array}$ \\
\hline Lau et al. & 2012 & China & $\begin{array}{c}\text { Estudo } \\
\text { experimental }\end{array}$ & Ratos & $\begin{array}{c}\text { Astragali Radix, } \\
\text { Rehmanniae Radix. }\end{array}$ & $\begin{array}{l}\text { Extrato das plantas utilizadas } \\
\text { juntamente. Topicamente. }\end{array}$ \\
\hline Suntar et al. & 2012 & Turquia & $\begin{array}{c}\text { Estudo } \\
\text { Experimental }\end{array}$ & Ratos & $\begin{array}{l}\text { Pinus brutia Ten.", } \\
\text { Pinus halepensis Mill. } \\
\text { Pinus nigra Arn", } \\
\text { Pinus pinea L., } \\
\text { Pinus sylvestris L“. }\end{array}$ & $\begin{array}{l}\text { Óleo essencial das plantas } \\
\text { aplicado topicamente. }\end{array}$ \\
\hline Akkol et al. & 2012 & Turquia & $\begin{array}{c}\text { Estudo } \\
\text { experimental }\end{array}$ & Ratos & $\begin{array}{c}\text { Ranunculus pedatus } \\
\text { Waldst., Ranunculus } \\
\text { constantinapolitanus (DC.)"* }\end{array}$ & $\begin{array}{c}\text { Extrato metanólico das } \\
\text { plantas aplicado topicamente. }\end{array}$ \\
\hline Güvenç et al. & 2012 & Turquia & $\begin{array}{c}\text { Estudo } \\
\text { experimental }\end{array}$ & Ratos & $\begin{array}{l}\text { Michauxia nuda (root), } \\
\text { Michauxia tchihatchewii } \\
\text { Fisch. \& CA Mey. }\end{array}$ & $\begin{array}{c}\text { Pomada com extrato } \\
\text { metanólico das plantas } \\
\text { topicamente. }\end{array}$ \\
\hline
\end{tabular}

continua...

Rev. Bras. PI. Med., Campinas, v.16, n.3, p.628-636, 2014. 
Quadro 2. Estudos clínicos e experimentais com plantas medicinais na cicatrização de feridas. Brasil. 2013.

\begin{tabular}{|c|c|c|c|c|c|c|}
\hline Wang et al. & 2013 & Alemanha & $\begin{array}{c}\text { Estudo } \\
\text { experimental } \\
\text { in vitro }\end{array}$ & $\begin{array}{c}\text { Células de pele } \\
\text { humana }\end{array}$ & $\begin{array}{c}\text { Radix et Rhizoma } \\
\text { Notoginseng, Radix } \\
\text { Angelicae Sinensis (Oliv.) } \\
\text { Diels, } \\
\text { Lonicerae japonicae Flos, } \\
\text { Paeonia suffruticosa Andr. }\end{array}$ & $\begin{array}{c}\text { Extrato hidroalcoólico das } \\
\text { plantas separadamente. }\end{array}$ \\
\hline Joshi et al. & 2013 & Índia & $\begin{array}{c}\text { Estudo } \\
\text { experimental }\end{array}$ & Ratos & $\begin{array}{c}\text { Albizzia lebbeck Benth. } \\
\text { Extrato etanólico da raiz } \\
\text { aplicado topicamente. }\end{array}$ \\
\hline Gilani et al. & 2013 & Paquistão & $\begin{array}{c}\text { Estudo } \\
\text { experimental }\end{array}$ & Coelhos & Trachyuspermum AMMl & $\begin{array}{c}\text { Creme com o óleo essencial } \\
\text { da planta a 2,86\%. }\end{array}$ \\
\hline
\end{tabular}

"Plantas que não apresentaram resultado positivo na cicatrização de feridas, no referido estudo.

popularmente conhecidas como cicatrizantes, por meio de estudos experimentais e clínicos. Para a classificação do tipo de estudo, utilizou-se a definição de Polit et al. (2004).

Analisando os dados emergentes na presente revisão, e enfatizando o quadro demonstrado, podemos concluir que a maioria dos estudos realizados é experimental, por meio de animais, geralmente ratos, em laboratório, com apenas dois estudos de cunho clínico randomizado.

Nesta pesquisa bibliográfica, os países que merecem destaque por pesquisarem sobre os efeitos de plantas medicinais na cicatrização são o Brasil, com 13 publicações, a Índia com 11, os Estados Unidos com cinco, Cuba com quatro, Turquia com três estudos, China, Peru, Japão e Tailândia com duas publicações, e os demais países citados aparecem com apenas um estudo. Quanto ao período de tempo compreendido nesta revisão, entre os anos de 1993 e 2013, destaca-se o período entre 1994 e 1999, totalizando 21 artigos publicados.

Quando nos referimos ao contexto brasileiro das pesquisas com plantas medicinais, não se pode deixar de mencionar alguns avanços alcançados nas últimas décadas. É o caso do extrato aquoso de Stryphnodendron adstringens (Mart.) Coville, o qual é tradicionalmente utilizado no Brasil como cicatrizante. Estudo clínico com 27 pacientes, conduzido por seis meses, teve por objetivo avaliar a eficácia de um medicamento na forma de pomada, contendo três por cento ( $3 \%$ ) de fitocomplexo fenólico de barbatimão na cicatrização de úlceras de decúbito. Durante a realização do estudo, $100 \%$ das lesões tratadas com o medicamento, cicatrizaram completamente. Esta planta é base para a produção de um fitoterápico já vendido nas farmácias brasileiras (Minatel et al, 2010).

Estudos sobre a planta Calendula officinalis L., Coronopu didymus (L.) Smith e Aloe Vera (L.) Burm. f., também são realizados no país, contribuindo para a comprovação das ações de reepitelização promovidas pela utilização dos seus extratos (Faleiro et al, 2009; Nitz et al, 2006).

Outra contribuição brasileira importante é a pesquisa realizada com a planta Anacardium occidentale L., sendo o seu efeito avaliado em ratos. A avaliação histopatológica do grupo tratado com POLICAJU demonstrou a presença de tecido de granulação fibrovascular no sexto dia pósoperatório, sugerindo a possível utilização clínica da emulsão, contendo seu polissacarídeo (Schirato et al, 2006).

A planta Crajiru (Arrabidaea chica Verlot.), também vem sendo estudada. Em vista da grande utilização popular, estudos in vitro e in vivo buscaram corroborar as ações cicatrizantes do extrato de suas folhas, obtendo resultados positivos (Jorge et al, 2008; Aro et al, 2013). Esta planta está na relação nacional de espécies medicinais de interesse ao SUS e já conta com um medicamento sendo desenvolvido com alto teor cicatrizante, para ulcerações diabéticas (Brasil, 2009).

Desta forma, um total de 52 plantas medicinais e um composto de ervas foram estudados experimentalmente ou clinicamente, quanto aos seus efeitos no auxílio do processo de cicatrização, algumas delas com mais de um estudo para comprovação. Verificou-se que, destas, 46 apresentaram potencial elevado de cicatrização de feridas, através de experimentação em ao menos um estudo, totalizando $88,5 \%$ e podendo ser utilizadas como terapia em processos de cura de feridas e processos inflamatórios.

Apenas seis plantas $(11,5 \%)$ não tiveram resultados positivos quanto aos seus efeitos cicatrizantes, sendo elas a Aloe vera (L.) Burm. f. associada à nitroglicerina, em um estudo de 1997 , o óleo puro de copaíba em seu primeiro teste, em 1998, o óleo essencial de Pinus brutia Ten., Pinus nigra Arn., Pinus sylvestris L., testados em ratos em 2012 e o Ranunculus constantinopolitanus (DC.), em estudo experimental, também em 2012. Considerando que a planta Aloe Vera (L.) Burm. f., foi a mais pesquisada nos últimos 20 anos, com 11 
estudos, elucidando seus potenciais cicatrizantes, e com 10 resultados positivos de eficácia.

Com isso, apesar do grande contingente de publicações em nível mundial, observa-se que o Brasil vem desenvolvendo pesquisas importantes para o avanço do conhecimento das propriedades medicinais das plantas utilizadas pela população. Outra constatação é que já existem programas e políticas que estimulam a inserção deste tipo de terapia no SUS, o que demonstra a busca pela oferta de um atendimento humanizado e integral.

Um dos avanços, além da Política Nacional de Plantas Medicinais (Brasil, 2006a) e a Política Nacional de Práticas Integrativas e Complementares (Brasil, 2006b), trata-se da RDC $n^{\circ} 10$, de 2010, que traz uma lista com 66 plantas medicinais que podem ser utilizadas e distribuídas pelos serviços de saúde, elucidando aspectos como dose, preparação e contraindicações. Dentre as plantas citadas, oito delas são indicadas para cicatrização de feridas, com comprovadas ações, sendo que algumas já foram citadas anteriormente no presente estudo. Dentre as principais, destacam-se a Anacardium occidentale L., Caesalpinia ferrea Mart., Casearia sylvestris Sw., Schinus terebinthifolia Raddi, Stryphnodendrom adstrigens (Mart.) Coville, Calendula officinalis L., Maytenus ilicifolia Mart. ex Reissek e Polygonum punctatum Elliott (Brasil, 2010a). Além disso, a planta Schinus terebinthifolius Raddi., com ações cicatrizantes, já é distribuída pelo sistema de saúde brasileiro na forma de medicamento fitoterápico, integrando a lista da Relação Nacional de Medicamentos Essenciais (RENAME) (Brasil, 2010b).

Com base no exposto, as plantas medicinais tornam-se alternativas de grande relevância para o processo de cicatrização de feridas, que começam a fazer parte da atenção à saúde brasileira, considerando que seu uso seja validado por estudos que afirmem seu potencial cicatrizante, o que sugere novos estudos de comprovação clínica, custos e benefícios, e a constante atualização acerca das publicações realizadas.

\section{REFERÊNCIA}

AKKOL, E.K. et al. Wound healing and anti-inflammatory properties of Ranunculus pedatus and Ranunculus constantinapolitanus: a comparative study. Journal of Ethnopharmacology, v.139, n.2, p.478-484, 2012.

ALVIM, N.A.T. et al. O uso de plantas medicinais como recurso terapêutico: das influências da formação profissional às implicações éticas e legais de sua aplicabilidade como extensão da prática de cuidar realizada pela enfermeira. Revista Latino-Americana de Enfermagem, v.14, n.3, p.316-323, 2006.

AQUINO, D. et al. de. Nível de conhecimento sobre riscos e benefícios do uso de plantas medicinais e fitoterápicos de uma comunidade do recife - PE. Revista de Enfermagem UFPE On Line, v.1, n.1, p.107-110, 2007.

ARO, A.A. et al. Effect of the Arrabidaea chica extract on collagen fiber organization during healing of partially transected tendon. Life Science, v.92, n.13, p.799807, 2013.

ATIBA, A. et al. The effect of Aloe vera oral administration on cutaneous wound healing in type 2 diabetic rats. Journal of Veterinary Medical Science, v.73, n.5, p.583-589, 2011.

BRASIL. Ministério da Saúde. Política Nacional de Plantas Medicinais e Fitoterápicos. Brasília: Ministério da Saúde, 2006a. 60 p.

BRASIL. Ministério da Saúde. Política Nacional de Práticas Integrativas e Complementares no SUS PNPIC-SUS. Brasília: Ministério da Saúde, 2006b. 92 p.

BRASIL. Ministério da Saúde. RENISUS - Relação de Plantas Medicinais de Interesse ao SUS. Brasília: Ministério da Saúde, 2009.

BRASIL. Ministério da saúde. Agência Nacional de Vigilância Sanitária. Resolução- RDC N 10 de 09 de março de 2010. Dispõe sobre a notificação de drogas vegetais. Brasília: Ministério da Saúde, 2010a.

BRASIL. Ministério da saúde. Secretaria de Ciência, Tecnologia e Insumos Estratégicos. Relação Nacional de Medicamentos Essenciais - RENAME. Brasília: Ministério da Saúde, 2010b.

BRITO, N.M.B. et al. Aspectos microscópicos da cicatrizaçäo de feridas cutâneas abertas tratadas com óleo de copaíba em ratos Gr. Revista Paraense de Medicina, v.13, n.1, p.12-17, 1999.

BRITO, N.M.B. et al. Efeitos do óleo de copaíba na cicatrizaçäo de feridas cutâneas abertas em ratos. Revista Paraense de Medicina, v.12, n.1, p.28-32, 1998.

CAMARGO, F.G. et al. Ação do extrato alcoólico de guaçatonga diluído e tamponado em subcutâneo de camundongo: Parte II: estudo histológico. Lecta-USF, v.14, n.1, p. 61-86, 1996.

CARVALHO, A. C. B.; SILVEIRA, D. Drogas vegetais: uma antiga nova forma de utilização de plantas medicinais. Brasília Médica, v.48, n.2, p.219-237, 2010.

CASTRO E SILVA, H.V. Ação da Stryphnodendron barbatiman sobre a cicatrização: estudo experimental em ratos. HB científica, v.3, n.1, p.77-79, 1996.

CAVALCANTE, A.R.S.M. et al. Análise tensional e morfológica da anastomose colônica na colite induzida por ácido acético a $10 \%$, em ratos Wistar, tratados com extrato aquoso de aroeira-do-sertão a $10 \%$ (Myracrodruon urundeuva fr. all.). Acta Cirúrgica Brasileira, v.20, n.2, p.180-186, 2005.

$\mathrm{CHAH}, \mathrm{K} . \mathrm{F}$. et al. Antibacterial and wound healing properties of methanolic extracts of some Nigerian medicinal plants. Journal of Ethnopharmacology, v.104, n.1-2, p.164-167, 2006.

CHITHRA, P. et al. Influence of Aloe vera on collagen characteristics in healing dermal wounds in rats. Molecular and Cellular Biochemistry, v.181, n.1-2, p.71-76, 1998.

CHITHRA, P. et al. Influence of Aloe vera on the healing of dermal wounds in diabetic rats. Journal of Ethnopharmacology, v.59, n.3, p. 195-201,1998. 
CHITHRA, P. et al. Influence of Aloe vera on the glycosaminoglycans in the matrix of healing dermal wounds in rats. Journal of Ethnopharmacology, v.59, n.3, p.179-186,1998.

CHITHRA, P.; et al. Influence of Aloe vera on collagen turnover in healing of dermal wounds in rats. Indian Journal of Experimental Biology, v.36, n.9, p.896901, 1998

DAVIS, R.H. et al. Aloe vera, hydrocortisone, and sterol influence on wound tensile strength and antiinflammation. Journal of the American Podiatric Medical Association, v. 82, .12, p. 614-621, 1994.

DAVIS, R.H. et al. Anti-inflammatory and wound healing activity of a growth substance in Aloe vera. Journal of the American Podiatric Medical Association, v.84, n.2, p.77-81, 1994.

DEVIENNE, K.F. et al. Das plantas medicinais aos fitofármacos. Revista Brasileira de Plantas Medicinais, v.6, n.3, p.11-14, 2004.

DOMÍNGUEZ SUÁREZ, A. et al. Efecto cicatrizante de extracto fluido de hojas de Siempre-viva. Revista cubana de plantas medicinais, v.6, n.1, p.16-18, 2001.

FALEIRO, C.C. et al. O extrato das folhas de babosa, Aloe vera na cicatrização de feridas experimentais em pele de ratos, num ensaio controlado por placebo. Natureza on line, v.7, n.2, p.56-60, 2009.

GALVÃO, C.M. et al. Revisão sistemática: recurso que proporciona a incorporação das evidências na prática da enfermagem. Revista Latino-Americana de Enfermagem, v.12, n.3, p.549-556, 2004.

GILANI G.R. et al. Preliminary evaluation of antimicrobial activity of cream formulated with essential oil of Trachyuspermum ammi. Journal of Pharmaceutical Sciences, v.25, n.5, p.893-896, 2013.

GONZÁLEZ-QUEVEDO, M.R. et al. Crema epitelizante de clorofila carotenos y vitaminas aplicada en heridas abiertas experimentales. Revista cubana de plantas medicinais, v.30, n.4, p.236-240, 2001.

GUILLERMO, F. et al. Efecto cicatrizante del tallo subterráneo de Peperomia scutellaefolia R. et P. en geles aplicados a Ratus norvergicus. Folia Dermatológica Peruana, v. 16, n.1, p.15-22, 2005.

GÜVENÇ, A. et al. Wound healing and antiinflammatory activities of the Michauxia L'Hérit (Campanulaceae) species native to Turkey. Journal of Ethnopharmacology, v.139, n.2, p.401-408, 2012.

HEGGERS, J.P. et al. Beneficial effect of Aloe on wound healing in an excisional wound model. Journal of Alternative and Complementary Medicine, v.2, n.2, p.271-277, 1996.

HEGGERS, J.P. et al. Effect of the combination of Aloe vera, nitroglycerin, and L-NAME on wound healing in the rat excisional model. Journal of Alternative and Complementary Medicine, v.2, n.2, p.149-153, 1997.

JORGE, M.P. et al. Evaluation of wound healing properties of Arrabidaea chica Verlot extract. Journal of Ethnopharmacology, v.118, n.3, p.361-363, 2008.

JOSHI, A. et al. The antimicrobial screening of a Barbadian medicinal plant with indications for use in the treatment of diabetic wound infections. Planta Medica, v.79, n.9, p.737-743, 2013

LAU, K.M. et al. Synergistic interaction between Astragali Radix and Rehmanniae Radix in a Chinese herbal formula to promote diabetic wound healing. Journal of Ethnopharmacology, v.141, n.1, p.250-256, 2012.

LI, S. et al. Prospective randomized controlled study of a Chinese herbal medicine compound Tangzu Yuyang Ointment for chronic diabetic foot ulcers: a preliminary report. Journal of Ethnopharmacology, v.133, n.2, p.543-550, 2011.

MANDELBAUM, S.H. et al. Cicatrização: conceitos atuais e recursos auxiliares. Parte I. Anais Brasileiros de Dermatologia, Rio de Janeiro, v.78, n.4, p 393-408, 2003.

MAQUART, F.X. et al. Triterpenes from Centella asiatica stimulate extracellular matrix accumulation in rat experimental wounds. European Journal of Dermatology, v.9, n.4, p.289-296, 1999.

MARTÍNEZ FERNÁNDEZ, C. et al. Efecto cicatrizante del extracto fluido de Romerillo (Bidens Alba Linné). Medicentro (Villa Clara), v.7, n.4, 2003.

MELO JÚNIOR, E.J. et al. Medicinal plants in the healing of dry socket in rats: microbiological and microscopic analysis. Phytomedicine, v.9, n.2, p.109-116, 2002.

MINATEL, D.G. et al. Estudo clínico para validação da eficácia de pomada contendo barbatimão (Stryphnodendron adstringens (Mart.) Coville) ${ }^{*}$ na cicatrização de úlceras de decúbito. Revista Brasileira de Medicina, v.67, n.7, p.250-256, 2010.

MORISAKI, N. et al. Mechanism of angiogenic effects of saponin from ginseng Radix rubra in human umbilical vein endothelial cells. British Journal of Pharmacology, v.115, n.7, p.1188-1193, 1995.

MOURA-LETTS, G. et al. In vivo wound-healing activity of oleanolic acid derived from the acid hydrolysis of Anredera diffusa. Journal of Natural Products, v.69, n.6, p.978-979, 2006.

MUKHERJEE, P.K. et al. Evaluation of in-vivo wound healing activity of Hypericum patulum (Family: hypericaceae) leaf extract on different wound model in rats. Journal of Ethnopharmacology, v.70, n.3, p.315-321, 2000.

MUKHERJEE, P.K.; SURESH, B. The evaluation of wound-healing potential of Hypericum hookerianum leaf and stem extracts. Journal of Alternative and Complementary Medicine, v.6, n.1, p.61-69, 2000.

NAGAPPA, A.N.; CHERIYAN, B. Wound healing activity of the aqueous extract of Thespesia populnea fruit. Fitoterapia, v.72, n.5, p.503-506, 2001.

NAYAK, B.S.; MOHAN, K. Influence of ethanolic extract of Jasminum grandflorum linn flower on wound healing activity in rats. Indian Journal of Physiology and Pharmacology, v.51, n.2, p.189-194, 2007.

NITZ, A.C. et al. Estudo morfométrico no processo de cicatrização de feridas cutâneas em ratos, usando: Coronopu didymus e Calendula officinali. Arquivos Catarinenses de Medicina, v.35, n.4, p.74-79, 2006.

NUZOV, B.G.; STADNIKOV, A.A. Effect of miliacin oil on healing of trophic ulcers. Patologicheskaia fiziologiia i eksperimentalnaia terapiia, n.4, p.55-56, 1994.

OLADEJO, O.W. et al. A comparative study of the wound healing properties of honey and Ageratum conyzoides. African Journal of Medicine \& Medical Sciences, v.32, n.2, p.193-196, 2003.

PARENTE, L.M.L. et al. Efeito cicatrizante e atividade antibacteriana da Calendula officinalis L. cultivada no 
Brasil. Revista Brasileira de Plantas Medicinais, v.11, n.4, p.383-391, 2009.

PARENTE, L.M.L. et al. Angiogenic activity of Calendula officinalis flowers L. in rats. Acta Cirúrgica Brasileira, v.26, n.1, p.19-24, 2011.

PARK, E.H.; CHUN, M.J. Wound healing activity of Opuntia ficus-indica. Fitoterapia, v.72, n.2, p.165-167, 2001.

POLIT, D.F. et al. Fundamentos da pesquisa em enfermagem: métodos, avaliação e utilização. 5 ed. Porto Alegre: Artmed, 2004.

PRICHOA, F.C. et al. Tissue injuries of wistar rats treated with hydroalcoholic extract of Sonchus oleraceus L. Brazilian Journal of Pharmaceutical Sciences, v.47, n.3, p.605-613, 2011.

ROBERTS, D.B.; TRAVIS, E.L. Acemannan-containing wound dressing gel reduces radiation-induced skin reactions in $\mathrm{C} 3 \mathrm{H}$ mice. International Journal of Radiation Oncology Biology Physics, v.32, n.4, p.1047-1052, 1995.

SAHA, K. et al. Wound healing activity of Leucas lavandulaefolia Rees. Journal of Ethnopharmacology, v.56, n.2, p.139-144, 1997.

SÁNCHEZ, C.A. et al. Effect of Calendula officinalis extract on postextraction alveolar bone formation in rabbits. Revista de Administração Contemporânea, v.52, n.2, p.89-94, 1995.

SCHIRATO, G.V. et al. O polissacarídeo do Anacardium occidentale L. na fase inflamatória do processo cicatricial de lesões cutâneas. Ciência Rural, v.36, n.1, p.149-154, 2006.

SERVIN, S.C.N. et al. Ação do extrato de Jatropha gossypiifolia L. (pião roxo) na cicatrização de anastomose colônica: estudo experimental em ratos. Acta Cirúrgica Brasileira, v.21, supl.3, p.89-96, 2006.

SILVA, D.M.; MOCELIN, K.R. O cuidado de enfermagem ao cliente portador de feridas sob a ótica do cuidado transcultural. Nursing (São Paulo), v. 9, n. 105, p. 8188, 2007.

SILVA, M.I. et al. A utilização da Pfaffia glomerata no processo de cicatrização de feridas da pele. Arquivos Brasileiros de Cirurgia Digestiva, v.23, n.4, p.228233, 2010.

SOMBOONWONG, J. et al. Therapeutic effects of Aloe vera on cutaneous microcirculation and wound healing in second degree burn model in rats. Journal Of The Medical Association Of Thailand, v.83, n.4, p.417425, 2000.

SUGUNA, L. et al. Effects of Centella asiatica extract on dermal wound healing in rats. Indian Journal of Experimental Biology, v.34, n.12, p.1208-1211, 1996.

SUNILKUMAR et al. Evaluation of topical formulations of aqueous extract of Centella asiatica on open wounds in rats. Indian Journal of Experimental Biology, v.36, n.6, p.569-572, 1998.

SÜNTAR, I. et al. Appraisal on the wound healing and anti-inflammatory activities of the essential oils obtained from the cones and needles of Pinus species by in vivo and in vitro experimental models. Journal of Ethnopharmacology, v.139, n.2, p.533-540, 2012.

TILLÁN CAPO, J.I. et al. Efecto cicatrizante de la crema de extracto etanólico de cera de caña. Revista cubana de plantas medicinais, v.9, n.2, 2004.

VISUTHIKOSOL, V. et al. Effect of Aloe vera gel to healing of burn wound a clinical and histologic study. Journal Of The Medical Association Of Thailand, v.78, n.8, p.403-409, 1995.

WANG, R. et al. Wound-healing plants from TCM: in vitro investigations on selected TCM plants and their influence on human dermal fibroblasts and keratinocytes. Fitoterapia, v.84, p.308-317, 2013. 